Schleicher, Stefan (2006): Umweltsteuern für innovative Wirtschaftsstrukturen, in: Kurswechsel, H. I, 87-95

Statistik Austria (2007): Einkommensbericht 2004 und 2005 gemäß Bezügebegrenzungsgesetz, Wien

Truger, Achim (2005): Einführung: Steuergerechtigkeit in der Defensive?, in: Truger, Achim (Hg.), Können wir uns Steuergerechtigkeit nicht mehr leisten?, Marburg, S. 7-I4

\title{
Zur Verteilung von Arbeitszeit und Ungleichheit. Eine geschlechts- spezifische Betrachtung im EU-Kontext \\ Gender Equality and Working Hours: The Obvious and the Hidden Connection
}

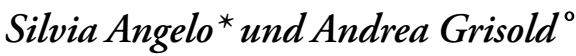

Dieser Artikel widmet sich einer Vorfrage, ja Vorbedingung für die Einkommens- und Vermögensverteilung: Innerhalb des Arbeitsmarktes wird die Frage der Arbeitszeiten untersucht. Die Frauenbeschäftigung in der Europäischen Union (EU) ist gestiegen, wie von Seiten der EU angestrebt, in vielen Ländern sogar stark. Es wird bei dieser Erfolgsmeldung allerdings gerne vergessen, dass dies zu einem großen Teil auf Teilzeitbeschäftigung basiert.

Der Blickpunkt der Diskussion zu Arbeitszeiten war in den letzten Jahren primär auf Aspekte der Deregulierung - bzw. seit den I99oer Jahren anders ausgedrückt: der Flexibilisierung - fokussiert. Die Tatsache, dass Arbeitszeiten allerdings nicht mehr generell, sondern individuell verkürzt werden, hat zu einer Neuverteilung der Arbeitszeiten zwischen den Geschlechtern geführt. Befürworter|innen dieser Entwicklung sehen darin eine logische Folge der zunehmenden Erwerbstätigkeit von Frauen: Da diese die Vereinbarkeit von Beruf und Familie meistern müssen, sind kürzere Arbeitszeiten funktional. Aufgrund dieser Entwicklung hat sich zwar gesamtgesellschaftlich statistisch betrachtet die Erwerbsarbeitszeit verringert, individuell gesehen kann aber gerade bei Frauen gezeigt werden, dass sich ihre Arbeitszeit insgesamt (bezahlte und unbezahlte Arbeit) nicht verringert hat. Wenn auch die Schlussfolgerung, dass sich damit die ungleiche Verteilung von Einkommen zwischen Frauen und Männern perpetuiert, als beinahe zu offensichtlich bezeichnet werden kann, sind doch Verteilungsfragen und Arbeitszeitfragen selten gekoppelte Themen.

\footnotetext{
* Wien.

o Wirtschaftsuniversität Wien.

(C) INTERVENTION 5 (I), 2008, 20-3I
} 
In allen europäischen Ländern sind es vorwiegend Frauen, die kürzer als die gesetzliche Normalarbeitszeit beschäftigt sind. Damit wird zum einen die Segmentierung des Arbeitsmarktes aufrecht erhalten, zum anderen die Einkommensschere zwischen den Geschlechtern nicht verringert, und zum dritten die bestehende traditionelle Arbeitsteilung im Bereich der unbezahlten Arbeit verfestigt.

In diesem Artikel wird der Frage nachgegangen, wie sich die ungleiche Verteilung von Erwerbsarbeitszeit auf "klassische« Verteilungsdisparitäten auswirkt. Die geschlechtsspezifische Verteilung der Erwerbsarbeitszeit wird für den EU-Raum analysiert, und die Gründe dieser Arbeitszeitwahl werden beleuchtet. Teilzeit dient in vielen europäischen Ländern zu einer Integration von Frauen in den Arbeitsmarkt; diese Integration ist allerdings "teuer" erkauft und bedeutet neben niedrigeren Aufstiegschancen auch geringeres Einkommen. Hier gilt es, die gesellschaftlichen Muster aufzuzeigen, die Frauen immer noch die Rolle der "Dazuverdienerinnen" zuschreiben. Wenn Frauenerwerbstätigkeit als Basis eigenständiger Existenzsicherung eher behindert wird, so kann von einer Sackgasse einer geschlechtsspezifischen Arbeitszeitpolarisierung gesprochen werden.

\section{Die Bedeutung der Teilzeitbeschäftigung für die Erwerbstätigkeit von Frauen}

Die Beschäftigung von Frauen ist in den letzten beiden Jahrzehnten EU-weit deutlich gestiegen. Nicht erst mit der Einführung des Lissabon-Zieles im Jahr 2000, das eine Beschäftigungsquote von EU-weit 6o Prozent bis 2010 vorsieht, sieht sich die EU mit einem deutlichen Aufholen der Frauen konfrontiert (siehe für alle Daten im Folgenden EU-Kommission 2007 und BALI Homepage): Die Beschäftigungsquote der Frauen lag 2006 bei 57,4 Prozent (EU-25) bzw. 58,4 Prozent für die EU-I5. Vergleicht man die Werte für die EU-I5 von 1995 und 2006, so betrug vor mehr als zehn Jahren die Differenz in den Beschäftigungsquoten noch rund 2I Prozentpunkte, während sie 2006 nur noch rund is Prozentpunkte ausmachte. ${ }^{\mathrm{I}}$ EUweit betrachtet ist im letzten Jahrzehnt die Männerbeschäftigung deutlich schwächer als jene der Frauen gestiegen. Allerdings sind die Unterschiede zwischen den einzelnen Mitgliedstaaten nach wie vor enorm: Der »employment gap «, also die Differenz in den Beschäftigungsquoten zwischen den Geschlechtern, reicht von rund 40 Prozentpunkten in Malta bis zu rund fünf Prozentpunkten in Schweden (Eurostat-Homepage Strukturindikatoren). Was die weitere Entwicklung betrifft, so sollte sich laut Prognosen der EU (DG ECFIN) die Beschäftigungsquote der Frauen bis 2025 auf 65 Prozent erhöhen und sich dann auf diesem Niveau stabilisieren (vgl. EU-Kommission 2007).

Die zunehmende Beschäftigungsquote von Frauen wird allerdings etwas relativiert, wenn nur die so genannten Vollzeitäquivalente betrachtet werden: Während die Beschäftigungsquote der EU-I5 von 49,7 Prozent 1995 auf 58,4 Prozent 2006 gestiegen ist, stiegen die Vollzeitäquivalente im gleichen Zeitraum nur von 42,3 Prozent auf 48,2 Prozent. Daran zeigt sich, dass Frauen verstärkt über Teilzeitbeschäftigung Zugang zum Arbeitsmarkt gefunden 
haben. Aber auch bei der Teilzeitbeschäftigung gibt es große Unterschiede zwischen den Mitgliedstaaten: Alle neuen EU-Mitgliedstaaten haben einen unterdurchschnittlichen Anteil an Teilzeitbeschäftigten, der EU-25-Durchschnitt beträgt 32,9 Prozent (bzw. 31,2 Prozent für die EU-27) bei Frauen, 7,7 Prozent bei Männern (EU-25 und EU-27). Mit Ausnahme von Dänemark und Schweden ist in den Ländern mit höherem Teilzeitanteil der Frauen die Teilzeitbeschäftigung von Männern im Vergleich verschwindend gering. So sind z. B. in Österreich über 40 Prozent der Frauen, aber nur rund sieben Prozent der Männer teilzeitbeschäftigt.

Auch wenn die Gründe für Teilzeitbeschäftigung - auf die im Folgenden nähereingegangen wird - in den europäischen Mitgliedstaaten unterschiedlich sind, so ist das Faktum, dass immer mehr Menschen kürzer als die Normalarbeitszeit beschäftigt sind, ein europäischer Trend. Dieser Trend war in vielen Ländern maßgeblich dafür verantwortlich, dass die Erwerbsbeteiligung von Frauen in den letzten Jahren angestiegen ist. Wobei ein Blick auf den Ländervergleich zeigt: Es gibt auch Länder, die ein hohes Beschäftigungsniveau von Frauen nicht über eine hohe Teilzeitquote erreicht haben (vgl. Abbildung I).

Abbildung I zeigt deutlich die Unterschiede zwischen den EU-Mitgliedstaaten hinsichtlich der Integration von Frauen in das Erwerbsleben. Im Wesentlichen spiegelt das Bild die vier "Modelle« der europäischen Wohlfahrtsstaaten wider (vgl. Guger/Aiginger 2005). ${ }^{2}$ Das skandinavische Modell (Schweden, Finnland, Norwegen, Dänemark und eingeschränkt auch die Niederlande), das korporatistische Modell (Deutschland, Österreich, Belgien und zum Teil Italien), das liberale Modell (Irland, Großbritannien) und das mediterrane Modell (Spanien, Portugal, Griechenland, zum Teil Italien).

Zwischen den beiden Polen - Niederlande und Bulgarien - finden sich die EU-Mitgliedstaaten wieder. Auf Basis der zitierten Modelle verlaufen die Unterschiede zwischen Ländern mit einer hohen (skandinavisches Modell) und jenen mit einer niedrigen Frauenbeschäftigung (mediterranes Modell). Erweitert um den Teilzeitanteil zeigt z. B. Finnland, dass eine hohe Beschäftigung von Frauen mit Vollzeiterwerbstätigkeit gekoppelt sein kann. Dort hat Teilzeitbeschäftigung fast keine Tradition und hat erst in den letzten Jahren etwas an Terrain gewonnen.

Umgekehrt sind es viele südliche Nachbarstaaten, die ein geringes Teilzeitniveau bei traditionell niedrigen Beschäftigungsquoten aufweisen. Spanien, Griechenland und Italien haben immer zu den europäischen Ländern mit niedriger Erwerbsbeteiligung von Frauen gehört. Allerdings hat sich auch hier in den letzten Jahren einiges verändert; so ist z. B. in Spanien in den letzten zehn Jahren ein enormer Anstieg der Frauenbeschäftigung festzustellen (von rund 33 Prozent 1996 auf rund 53 Prozent 2006), und auch die Teilzeitquote steigt kontinuierlich.

2 Wobei in dieser von Guger und Aiginger verwendeten Systematik, die auf Gosta Esping-Anderson zurückgeht, die neuen Mitgliedstaaten nicht berücksichtigt sind. Diese finden sich in Versatzstücken in den bestehenden Modellen wieder und sind sicherlich nicht in »einer" Kategorie zusammenfassbar. 


\section{Abbildung I: Beschäftigungsquoten und Teilzeitquoten Frauen, 2006}

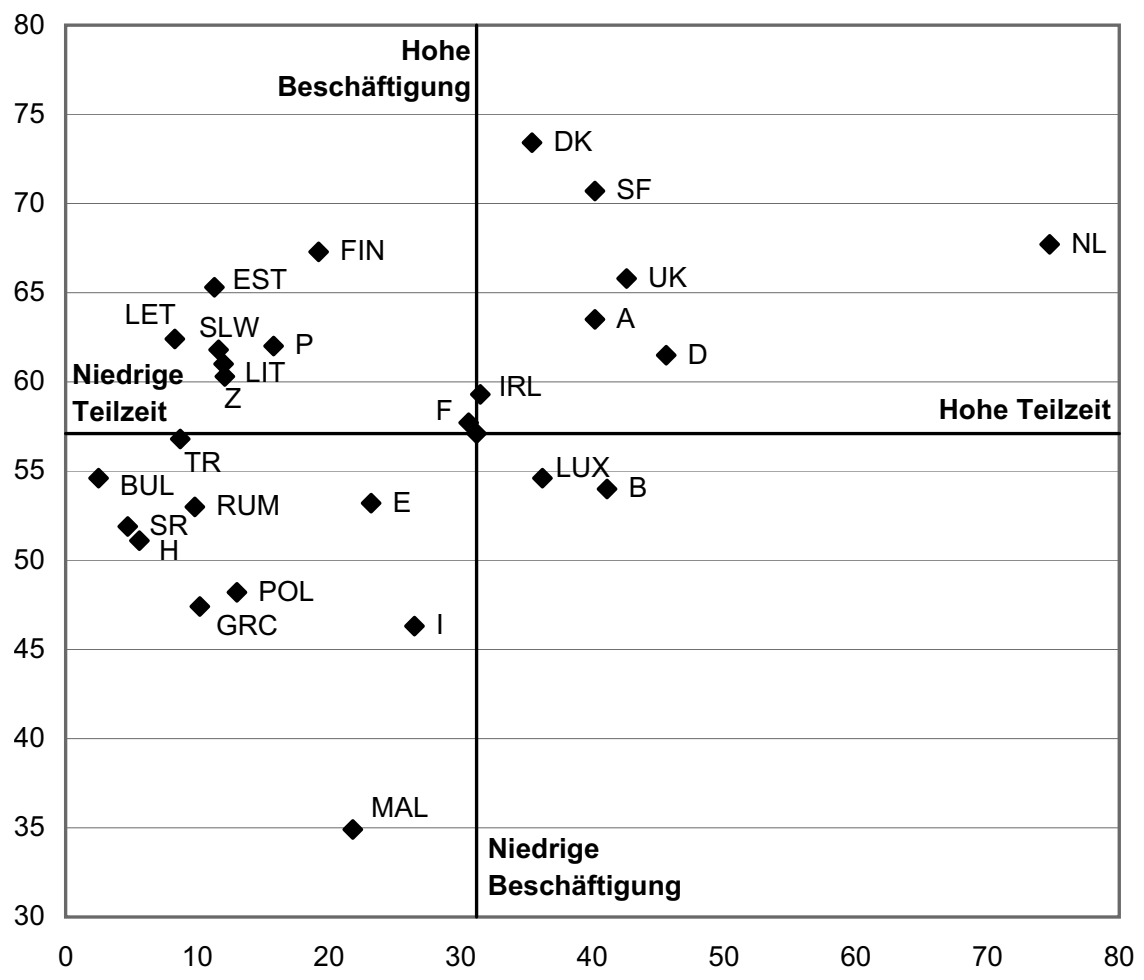

I Der Achsenschnittpunkt markiert den EU-27-Durchschnitt.

Quelle: ELIS Datenbank, eigene Darstellung

Was die neuen Mitgliedstaaten betrifft, so sind eindeutige Aussagen aufgrund der unterschiedlichen Bewältigung des ökonomischen und politischen Aufholprozesses hin zu den»alten" Mitgliedstaaten schwierig zu treffen. Aus Abbildung I wird ersichtlich, dass die drei baltischen Länder sowie Slowenien und Zypern ein hohes Maß an Frauenbeschäftigung aufweisen, wohingegen insbesondere in den bevölkerungsmäßig großen neuen Mitgliedstaaten eine unterdurchschnittliche Frauenbeschäftigung vorzufinden ist. In vielen dieser Länder waren Frauen bis zum »Fall des eisernen Vorhangs" deutlich stärker in den Arbeitsmarkt integriert, als sie es heute sind. Darüber hinaus hat der Anpassungsprozess an westliche Standards auch seine »Opfer" gefordert, die insbesondere in den Folgen des Abbaus staatlicher Strukturen bestanden haben. Diesen Abbau haben - nicht zuletzt aufgrund der klassischen Arbeitsteilung - besonders Frauen zu spüren bekommen. Teilzeitarbeit hat - betrachtet man Abbildung I unter diesem Aspekt - offenbar in vielen dieser Länder kaum eine Tradition und nimmt erst in den letzten Jahren sicherlich auch aufgrund des größer werdenden Dienstleistungssektors zu (vgl. Europäische Kommission 2007, Datenteil). 


\section{Gründe für Teilzeitbeschäftigung}

Die Gründe für die steigende Beteiligung von Frauen am Arbeitsmarkt sind zum einen in sich ändernden gesellschaftlichen Werten und zum anderen in ökonomischen Verschiebungen der Wirtschaftsstrukturen zu suchen. Wie immer bei solchen Entwicklungen, die sich aus gesellschaftlichen und wirtschaftlichen Veränderungen ergeben, gibt es auch hier Wechselwirkungen, ist das eine nicht ohne das Andere erklärbar. Nichts desto weniger lassen sich bestimmte Entwicklungen in der Arbeitswelt beobachten, die traditionell stärkere Anreize auf Frauen gehabt haben, ihr Arbeitskräfteangebot am Markt anzubieten (allerdings in einem zeitlich eingeschränkteren Umfang als Männer): In einem stark segregierten Arbeitsmarkt mit typischen Frauen- und Männerberufen haben Frauen durch die Ausweitung des Dienstleistungssektors Zugang zu bezahlter Arbeit erhalten. Die Segregation am Arbeitsmarkt wird durch diese Entwicklung natürlich noch verstärkt.

Warum Teilzeitarbeit für Frauen so attraktiv ist, hängt im Wesentlichen mit ihrer gesellschaftlichen Rolle als die primär für die Kinderbetreuung Zuständigen zusammen. Das Vorhandensein, die Anzahl und das Alter von Kindern bestimmen das Ob und das Wieviel der Frauenbeschäftigung (vgl. Europäische Kommission 2005). Dieses Rollenmuster unterliegt natürlich in der europäischen Betrachtung großen Schwankungen: Traditionell weisen die skandinavischen Länder wesentlich höhere Erwerbsquoten von Müttern auf als die südlichen Mitgliedstaaten.

Als Gründe dafür werden in erster Linie institutionelle Faktoren - die Möglichkeit der Vereinbarkeit von Beruf und Familie und das Steuersystem - angeführt. Während es in einigen Ländern Europas absolut üblich ist, Kinder extern zu betreuen, stößt dies in anderen Ländern auf Unverständnis. Auf Basis einer Untersuchung der EU-Kommission aus dem Jahr 2005 ergibt sich ein sehr uneinheitliches Bild, was die Versorgung mit Kinderbetreuung seinrichtungen betrifft (vgl. Europäische Kommission 2005). Während die Betreuungsquote der Null- bis Dreijährigen im flämischen Teil Belgiens 8I Prozent beträgt, in Dänemark bei 56 Prozent und in Schweden bei 4I Prozent liegt, weisen Länder wie Deutschland, Italien, Griechenland und Österreich Werte unter zehn Prozent auf. Demgegenüber liegt die Betreuungsquote der Drei- bis Sechsjährigen wesentlich höher: Hier schwankt der Großteil der Mitgliedstaaten zwischen 80 Prozent und 98 Prozent - anders die baltischen Staaten, die Tschechische Republik, Griechenland, Portugal, Slowenien, Slowakei und Finnland, die Werte unter 80 Prozent aufweisen (vgl. Europäische Kommission 2005).

Neben dem Angebot an Kinderbetreuung sind es aber natürlich auch noch andere Faktoren, wie die Möglichkeit des Karenzurlaubes oder flexibler Arbeitszeiten, die insbesondere bei Müttern die Frage des Einstiegs, Verbleibs, aber auch des Ausmaßes an Arbeitszeit beeinflussen.

Dass institutionelle Faktoren, wie etwa das Steuersystem, einen Einfluss auf das Erwerbsverhalten von Frauen haben, wird in letzter Zeit wieder verstärkt diskutiert. Aktuelle Beispiele einer öffentlichen Diskussion zu diesem Thema sind die jüngsten Vorschläge der Ökonomen Alesina / Ichino (2007), die eine positive Diskriminierung von Frauen durch eine Differenzierung der Steuersätze zwischen den Geschlechtern vorschlagen. In Österreich war 
es die im Herbst von der Österreichischen Volkspartei in die politische Diskussion eingebrachte Idee zu einem so genannten Familiensplitting. Dieses Modell sieht die gemeinsame Besteuerung des Familieneinkommens vor. Dies hätte allerdings - vor dem Hintergrund der hohen Einkommensunterschiede zwischen Frauen und Männern - negative Anreize auf das Erwerbsverhalten von Frauen.

Während der Großteil der europäischen Länder dieser Tatsache mit einem Individualsteuersystem Rechnung trägt, gibt es in einigen Mitgliedstaaten noch die so genannte Haushaltsbesteuerung bzw. Versatzstücke derselben (z. B. Ehegattensplitting in Deutschland oder Familiensplitting in Frankreich). Allerdings müssen bei einem Vergleich dieser Systeme immer auch die Betreuungsleistungen eines Landes mitberücksichtigt werden: So sind z. B. in Frankreich die monetären Leistungen für Familien im Gegensatz zu Österreich relativ gering, während vergleichsweise viel Geld in Sachleistungen (Kinderbetreuung von Kleinkindern und Ganztagsschule) fließt.

Diese Faktoren beeinflussen aber nicht nur die Frage, ob Frauen erwerbstätig sind, sondern auch, in welchem Ausmaß: So sind in Österreich und Deutschland zwar ähnlich viele Frauen mit Kindern über zwei Jahren berufstätig, allerdings liegt der Anteil jener Frauen, die in Vollzeit beschäftigt sind, in Österreich um Ioo Prozent über jenem in Deutschland (vgl. Dearing et al. 2007). In diesem Fall wird dies insbesondere mit den unterschiedlichen Anreizwirkungen der Steuersysteme (individuellem versus familienbasiertem Steuersystem) bzw. der Elternurlaubsvereinbarungen erklärt (vgl. ebd.).

\section{Fakten zur Teilzeitbeschäftigung}

In einer in Österreich durchgeführten repräsentativen Befragung geben Frauen vorrangig familiäre Gründe für Teilzeitarbeit an, ${ }^{3}$ bei (den wenigen) in Teilzeit arbeitenden Männern sind es gesundheitliche Gründe oder eine laufende Ausbildung (vgl. BMSG 2003). Frauen gaben am häufigsten an, sich dem Haushalt, den Kindern oder der Pflege von Angehörigen widmen zu müssen (25 Prozent), manche, durch diese Tätigkeiten bereits überlastet zu sein (elf Prozent) - je älter eine Frau ist, desto häufiger wird dieses Argument genannt (BMSG 2003: 42 f.). Auch der Wunsch des Ehepartners oder der Familie spielt eine Rolle (13 Prozent). Sieben Prozent geben an, es fehle an geeigneter institutioneller Kinderbetreuung.

Dabei ist es natürlich wichtig zu betrachten, ob Teilzeitarbeit freiwillig oder nur aufgrund des Mangels an Alternativen gewählt wurde. Ob die zunehmende Verbreitung von Teilzeitarbeit angebots- oder nachfrageseitig bestimmt ist, ist nicht monokausal zu beantworten. Zweifelsohne sind es die Interessen der Arbeitgeber|innen hinsichtlich größerer Flexibilisierung, die hier berücksichtigt werden (vgl. Bergmann et al. 2003), aber auch die in Teilzeit beschäftigten Personen geben positive Begründungen für diese Arbeitszeitwahl an (Zeitautonomie und -flexibilität), wie aus den Interviews in Bergmann et al. (2003) klar hervorgeht.

3 Interessant dabei ist noch festzuhalten, dass die Gründe gegen eine Aufnahme der Erwerbstätigkeit im Wesentlichen dieselben sind. 
Auf EU-Ebene wird diese Frage wie folgt beantwortet (vgl. zum Folgenden Guger/ Aiginger 2005: I8 ff. $)^{4}$ : Während in Finnland rund 33 Prozent aller in Teilzeit beschäftigten Frauen angeben, dass sie keine Vollzeitbeschäftigung gefunden haben, sind es in den Niederlanden nur 2,5 Prozent. Diesen Anteil könnte man als »klassische unfreiwillige Teilzeitarbeit « bezeichnen. Das heißt, in den Niederlanden arbeitet der Großteil der Frauen freiwillig Teilzeit. Allerdings kommt es natürlich darauf an, ob andere Gründe - wie z. B. die Notwendigkeit der Versorgung minderjähriger oder älterer Personen - auch als »freiwillig « definiert werden. Vor dem Hintergrund einer solchen - etwas differenzierten - Fragestellung sehen die Ergebnisse etwas anders aus: In Österreich geben rund elf Prozent der teilzeitbeschäftigten Frauen an, dass sie keine Vollzeitbeschäftigung gefunden haben (sie sind also klassisch unfreiwillig teilzeitbeschäftigt), allerdings geben rund 40 Prozent der teilzeitbeschäftigten Frauen auch an, dass sie aufgrund von Betreuungspflichten nicht vollzeiterwerbstätig sind. In Finnland betrifft dies nur rund sieben Prozent. Der Anteil der Frauen, die in Teilzeit arbeiten, da sie Betreuungspflichten wahrnehmen (müssen), ist in den Ländern des korporatistischen und des liberalen Modells am größten. Nicht zuletzt hängt dies mit den zuvor angesprochenen fehlenden Kinderbetreuungsmöglichkeiten insbesondere für Kleinkinder zusammen.

Als ein weiteres Faktum der Teilzeitarbeit darf nicht außer Acht gelassen werden, dass sie nur in den geringsten Fällen berufshierarchisch höher angesiedelt ist. Für Österreich wird dies in einer Befragung von Arbeitgeber|inne|n hauptsächlich damit begründet, dass Probleme mit der Arbeitsorganisation befürchtet werden. 5 Das Qualifikationsniveau hingegen ist bei Teilzeitbeschäftigten keineswegs geringer als bei Vollzeitbeschäftigten (vgl. Bergmann et al. 2003), was bedeutet, dass in Teilzeit beschäftigte Frauen oft Arbeiten unter ihrem Qualifikationsniveau verrichten.

All diese zitierten Zusammenhänge und Gründe für Teilzeitarbeit werden auch in empirischen Untersuchungen belegt:

"Econometric analyses using micro data were used to investigate the determinants of an individual's decision to work part-time [...]. The analyses reveal that preferences for individuals' part-time work depend on a number of factors, such as gender, education, household composition (e.g. number of children), government policies (e.g. taxation, child benefits/childcare subsidies) and the country's characteristics that may reflect cultural factors as well as national differences in labour market institutions." (Europäische Kommission 2007: I28)

Dies ist vor der Folie der überwiegenden Erbringung von unbezahlter Arbeit durch Frauen zu sehen, auf die im Folgenden eingegangen wird.

4 Da diese Detail-Befragungen weder jährlich noch in allen Mitgliedstaaten durchgeführt werden, wird hier auf die Studie von Guger /Aiginger (2005) und die Daten aus dem Jahr 200 I verwiesen. 5 Bergmann et al. (2003) finden in ihren Interviews mit Unternehmer|inne|n auch, dass das Kostenargument (Teilzeitbeschäftigte seien zu teuer im Vergleich zu Vollzeitarbeitsplätzen) von geringer Bedeutung ist. 


\section{Bezablte versus unbezahlte Arbeit}

Betrachten wir Österreich als Fallbeispiel, so zeigt eine Mikrozensus-Sondererhebung (vgl. BMSG 2003): Frauen weisen mit durchschnittlich 45,2 Stunden pro Woche (für Erwerbsarbeit, Haushalt und Kinderbetreuung) eine höhere Arbeitsbelastung auf als Männer mit 35, I Stunden. In allen Altersklassen arbeiten Frauen mehr Stunden als Männer. Dies ist besonders auf Nicht-Erwerbsarbeit (Kinderbetreuung und Haushaltsarbeit) zurückzuführen. In der Zeit zwischen 1995 (erstmalige Durchführung einer Mikrozensuserhebung zu diesem Thema) und 2002 gab es dabei keine grundlegende Änderung der geschlechtsspezifischen Arbeitsteilung.

Wenn wir auch glauben zu wissen, wie die Arbeitsteilung zwischen den Geschlechtern funktioniert, so überrascht bisweilen dennoch die Stärke dieser Unterschiede. Von der Arbeitszeit der Frauen entfallen fast zwei Drittel (6I,8 Prozent) auf Haushalt und Kinderbetreuung, bei Männern nur ein Fünftel (20,5 Prozent) (vgl. BMSG 2003). Dass Frauen verstärkt für die Haushaltsführung zuständig sind, ist im Falle einer Haupt- oder Alleinerwerbstätigkeit des Mannes nicht weiter verwunderlich. Aber auch bei erwerbstätigen Paaren führen mehrheitlich Frauen den Haushalt. ${ }^{6}$ Bei Kindererziehung ist der Männeranteil zwar höher als bei Haushaltstätigkeiten, Mütter wenden aber immer noch mehr an Zeit dafür auf. Da die Haushaltsführung partnerschaftlicher ist, je jünger die Paare sind (ebd: 28 ff.), kann auf eine langsame Änderung der geschlechtsspezifischen Arbeitsverteilung geschlossen werden. Ob diese auch nachhaltig ist, werden erst Untersuchungen in der Zukunft zeigen können.

Eine Studie zu den Arbeitszeit-Unterschieden zwischen ausgewählten europäischen Ländern und den USA (vgl. Burda et al. 2006) kommt zu dem Schluss, dass die Summe der wöchentlichen marktvermittelten (Erwerbs-)Arbeit und der Haus- und Familienarbeit (sekundäre Aktivitäten genannt, sie substituiert Arbeit, die auch marktvermittelt zugekauft werden könnte, und wird selbst nicht entlohnt) in den untersuchten Ländern zeitpunktbezogen über die Geschlechter gleich verteilt sind. ${ }^{7}$ Diese auffällige Ähnlichkeit die Gesamtarbeitszeit, d. h. marktvermittelte und nicht marktvermittelte Arbeit, ist innerhalb eines Landes bei Frauen und Männern annähernd gleich ${ }^{8}$ - darf nicht darüber hinwegtäuschen, dass große Unterschiede zwischen den Ländern existieren.

Für die Zeitverteilung zwischen den Geschlechtern kommen Burda et al. (2006) zu folgenden Schlüssen: Frauen haben weniger Freizeit als Männer, sie bringen viel mehr Zeit für household production auf, etwas mehr für tertiäre Aktivitäten. Geschlechtsspezifische

6 Berufstätige Mütter, die mit ihrem Partner leben, haben eine Gesamtarbeitsbelastung von 7I,8 Stunden (!) pro Woche.

7 Als Daten wurden Zeitbudgeterhebungen, die auf nationaler Ebene durchgeführt wurden, verwendet (Burda et al. 2006: Io ff.).

8 Es sei hier aber angemerkt, dass bei den verwendeten Datensätzen große Unterschiede bestehen: So ist z. B. in den US-Daten 1985 die Rubrik "Personal care outside the house « unter tertiary work rubriziert, 2003 hingegen werden "non-household care activities« unter leisure gereiht. 
Unterschiede sind in den USA etwas geringer als in europäischen Staaten; ein Umstand, der auf die geringere Differenz bei marktvermittelter Arbeit zurückgeführt wird, teilweise aber auch auf die größeren Ähnlichkeiten im nicht-marktvermittelten Zeitverhalten. Generell wird die höhere marktvermittelte Arbeit der Männer durch ihre geringere Haushaltsarbeit ausgeglichen. Im Längsschnittsvergleich zeigt sich, dass die gesamte Arbeitszeit (bezahlte und unbezahlte Arbeit) in den I4 Jahren Differenz zwischen den untersuchten Erhebungen (I6 Fälle: vier Länder, zwei Jahre, zwei Geschlechter) deutlich zurückgegangen ist, die Mehrarbeit von Frauen (mit 75 Minuten pro Tag) gegenüber Männern aber annähernd gleich geblieben ist (Burda et al. 2006: I8). Auch für Österreich lässt sich dies zeigen. Hier entfallen bei Frauen auf jede entlohnte Arbeitsstunde 5I Minuten an unbezahlter Arbeit im Haushalt, bei Männern nur elf Minuten. Dieses Muster, das sich in allen entwickelten Volkswirtschaften zeigt, hat ganz klare verteilungspolitische Konsequenzen: Geringeres eigenständiges Einkommen, geringere soziale Absicherung, geringerer Wohlstand. Was bei funktionierenden Beziehungen innerhalb der Familie ausgeglichen werden kann (aber nicht zwangsläufig muss), ist bei einer Scheidungsrate von rund 5o Prozent (Beispiel Österreich) nicht mehr der Normalfall. So kann es kaum verwundern, dass Armut bzw. ein Armutsrisiko bei Frauen verstärkt auftreten.

\section{Arbeitszeit und Einkommensverteilung}

Die geschlechtsspezifische Differenz zwischen bezahlter und unbezahlter Arbeit hat eindeutige Effekte in der Einkommensverteilung: Frauen arbeiten weniger marktvermittelt, d.h. mit direkter, monetärer Entlohnung verbunden, sondern mehr im Rahmen sekundärer Aktivitäten, die nicht direkt vergütet werden, in vielen Ländern auch nicht oder in geringerem Maße zu einem Anspruch auf Transferleistungen führen.

Eine simple Unterteilung in marktvermittelte Arbeit und andere Zeit - die dann ja meist als Nicht-Arbeit klassifiziert wird - ist nicht hilfreich, wenn es um die Verteilung von Arbeit an sich geht, sehr wohl aber, wenn es um die Verteilung von Arbeitseinkommen geht (dies ohne Transferleistungen, die in vielen Ländern aber auch größtenteils an frühere marktvermittelte Arbeitsleistungen gekoppelt sind). Die Ungleichverteilung zwischen bezahlter und unbezahlter Arbeit wirkt sich selbstverständlich in der Einkommensverteilung der Geschlechter aus: Der Einkommensunterschied ist erstaunlich persistent, sowohl in der Europäischen Union (vgl. Plantenga/Remery 2006) als auch in Österreich (vgl. Böheim et al. 2005). 2005 betrug in Österreich das Frauen-Medianeinkommen 67,I Prozent desjenigen der Männer. Dieser Einkommensunterschied ist jedoch keineswegs nur auf die höhere Teilzeitquote bei Frauen zurückzuführen. Arbeitszeitbereinigt beträgt das Frauen-Medianeinkommen 86,2 Prozent desjenigen der Männer (vgl. Guger/Marterbauer 2007). ${ }^{9}$

Die Erfassung der Einkommensunterschiede kann noch eine Stufe weniger aggregiert durchgeführt werden: Bei den unterschiedlichen Stundenlöhnen. In der EU verdienten Frauen 2002 auf Bruttostundenlohnbasis um fast 25 Prozent weniger als Männer. Dieser 
sehr große Einkommensunterschied will genauer analysiert werden. Eine komparative Studie der Einkommensunterschiede in 30 europäischen Ländern (vgl. Plantenga/ Remery 2006) zeigt große Unterschiede zwischen den einzelnen Ländern: von elf Prozent Einkommensunterschied in Slowenien bis zu 30 Prozent in Großbritannien erstreckt sich die Bandbreite. ${ }^{10}$ Wenn als Begründung dafür angeführt wird, dass Frauen mehr Schwierigkeiten in Ihren Karrieremöglichkeiten haben und weniger befördert werden, so ist dies einerseits ein generelles Phänomen, über den hohen Teilzeitanteil der Frauen aber noch einmal intensiver argumentierbar. Interessant ist auch, dass der Einkommensunterschied viel geringer ist, wird die Gruppe der Berufseinsteiger|innen untersucht. Weiters ist er bei verheirateten Arbeitnehmer|inne|n höher als bei Ledigen.

Innerhalb der EU wird die Reduzierung des Einkommensunterschiedes zwischen den Geschlechtern als ein wichtiges Thema der europäischen Politik identifiziert. So enthält die Europäische Beschäftigungsstrategie 2003 eine Aufforderung an die Mitgliedstaaten, »bis 2010 eine wesentliche Reduzierung [... ] zu erreichen« (vgl. Plantenga/ Remery 2006). Dabei wird immer von den Bruttostundenlöhnen als Grundlage ausgegangen, daher der Anstieg der Frauen-Teilzeitarbeit, der ja aus der Beschäftigungsoffensive der EU (und ihren Flexibilisierungsbestrebungen wie -erfordernissen der Arbeit) heraus erklärbar ist, vernachlässigt.

Die derzeitige Einkommenssituation von (vorwiegend weiblichen) Teilzeitbeschäftigten kann zusammenfassend wie folgt charakterisiert werden: Da diese verstärkt in Niedriglohnbereichen tätig sind, sind die Bruttostundenverdienste generell niedriger als bei Vollzeitbeschäftigung, dazu kommt noch der geringere Gesamtverdienst durch geringere Arbeitszeit. Bei einer schon vorfindbaren starken Einkommensdifferenzierung wie in Österreich (vgl. OECD 2007) führt das nicht nur zu geringem Erwerbseinkommen der Teilzeitbeschäftigten, sondern über das Äquivalenzprinzip setzt sich dies in der sozialen Sicherung, besonders auch bei den Pensionen und beim Arbeitslosengeld, fort.

\section{Resümee: Verteilung und Arbeitszeit}

Teilzeitarbeit ist weiblich und wird es in absehbarer Zeit auch bleiben. Dies trägt einerseits zur Integration von Frauen in den Arbeitsmarkt bei, ist aber auch eine Hürde für die Chancengleichheit, besonders, wenn es um die Verteilung von Einkommen geht. Teilzeitarbeit ist aber auch in den unteren Hierarchiestufen angesiedelt, weniger gut abgesichert und bietet kaum Aufstiegschancen respektive Weiterbildungsmöglichkeiten. Darüber hinaus verstärkt Teilzeitbeschäftigung von Frauen die bestehende Verteilung der überwiegenden Zuständigkeit der Frauen für Haushalt und Kindererziehung.

Die Neuverteilung der bezahlten Arbeit in den letzten Jahrzehnten kann in zwei Komponenten zerlegt werden: Die durchschnittliche Arbeitzeit pro Beschäftigtem bzw. Beschäf-

IO Dabei muss angemerkt werden, dass sich die dabei verwendeten Daten auf die Verdienststrukturerhebung 2002 beziehen, die nur Beschäftigte in der Privatwirtschaft beinhaltet. Wir wissen aber, dass Frauen im öffentlichen Sektor überrepräsentiert sind und dass der Einkommensunterschied dort geringer ist. Geisberger (2007) analysiert diese Daten für Österreich im Detail. 
tigter ist in den letzten 50 Jahren aufgrund kollektivvertraglicher bzw. gesetzlicher Regelungen ständig zurückgegangen. Im Gegensatz zu den vorangegangenen Perioden war dies ab den I980er Jahren allerdings primär Resultat individueller Arbeitzeitverkürzungen (d.h. Anstieg der Teilzeitbeschäftigung) und nicht kollektiver (d.h. tariflicher) Verkürzungen der Vollzeitarbeit. Gleichzeitig mit dem Anstieg der Teilzeitbeschäftigung ist auch die Erwerbsbeteiligung von Frauen gestiegen. Aufgrund dieser Entwicklung hat sich zwar statistisch gesamtgesellschaftlich betrachtet die Arbeitszeit verringert, individuell gesehen sind Frauen davon weniger betroffen, da sich ihre Arbeitszeit insgesamt (bezahlte und unbezahlte Arbeit) nicht verkürzt hat. Die Frage, die sich an die Entwicklung bei der Arbeitszeit knüpft, ist, ob »kollektivvertragliche Verkürzungen der Standardarbeitszeit tatsächlich durch die Ausbreitung von Teilzeitarbeit ersetzt werden « können (Lehndorff I998: 57I), ob es sich dabei also um ein Zukunftsmodell handelt. Verteilungstechnisch gesehen wäre es sinnvoller, nicht die individuelle, sondern die kollektive Arbeitszeit zu verkürzen.

»Unter gesellschaftlichen Bedingungen, die Frauenerwerbstätigkeit als Basis eigenständiger Existenzsicherung eher behindern (der britische Weg), droht eine Ausweitung der Teilzeitarbeit als Ersatz für weitere Verkürzungen der Standardarbeitszeit in der Sackgasse einer geschlechtsspezifischen Arbeitszeitpolarisierung.» (Lehndorff 1998: 575)

Aber diese Option der generellen Arbeitszeitverkürzung scheint aus der politischen und öffentlichen Debatte derzeit völlig verschwunden zu sein.

\section{Literatur}

Alesina, Alberto/Ichino, Andrea (2007): Gender Based Taxation, mimeo

BALI Homepage BMWA, URL: http://bali.bmwa.gv.at/

Bergmann, Nadja / Fink, Marcel/Graf, Nikolaus / Hermann, Christoph/Mairhuber, Ingrid / Sorger, Claudia/Willsberger, Barbara (2003): Qualifizierte Teilzeitbeschäftigung in Österreich. Bestandsaufnahme und Potenziale, Wien

BMSG (2003): Haushaltsführung, Kinderbetreuung, Pflege. Ergebnisse des Mikrozensus 2002, Wien

Böheim, René/Hofer, Helmut/Zulehner, Christine (2005): Wage Differences Between Men and Women in Austria: Evidence from 1983 and 1997, IZA Discussion Paper, Nr. 1554

Burda, Michael C. / Hamermesh, Daniel C. / Weil, Philippe (2006): Different but Equal: Total Work, Gender and Social Norms in EU and US Time Use, Paper presented at the Conference of the Fondazione Rodolfo Debenedetti, Portovenere, Italy, 27. April

Dearing, Helene/Hofer, Helmut/Lietz, Christine/ Winter-Ebmer Rudolf/Wrohlich, Katharina (2007): Why are Mothers Working Longer Hours in Austria Than in Germany? A Comparative Micro Simulation Analysis, IHS Reihe Ökonomie, Nr. 213, Wien

ELIS Homepage BMWA, URL: http://www.dnet.at/elis/

Europäische Kommission (2005): Reconciliation of Work and Private Life: A Comperative Review of Thirty European Countries, Brüssel: Europäische Kommission 
Europäische Kommission (2007): Employment in Europe 2007, COM(2007) 359 final, Luxemburg

Eurostat Homepage Strukturindikatoren, URL: http://epp.eurostat.ec.europa.eu/portal/page?_ pageid=II33,47800773,II33_47803568\&_dad=portal\&_schema=PORTA

Geisberger, Tamara (2007): Geschlechtsspezifische Lohn- und Gehaltsunterschiede, in: Statistische Nachrichten, Jg. 62, Nr. 7, S. 633-642

Guger, Alois/Aiginger, Karl (2005): Das europäische Gesellschaftsmodell, Wien

Guger, Alois / Marterbauer, Markus (2007): Langfristige Tendenzen der Einkommensverteilung in Österreich - ein Update. Die Verteilung von

Einkommen und Vermögen, WIFO Working Papers, Nr.307/2007

Lehndorff, Steffen (1998): Von der »kollektiven« zur »individuellen« Arbeitszeitverkürzung?, in: WSI Mitteilungen, Jg. 5I, Nr. 9, S. 569-579

OECD (2007): OECD Employment Outlook, Paris: OECD

Plantenga, Janneke/Remery, Chantal (2006): The Gender Pay Gap - Origins and Policy Responses. A Comparative Review of 30 European Countries, Luxemburg: Europäische Kommission

\section{Arme Schuldner - Reiche Schuldner? Haushaltsverschuldung und Geldvermögen privater Haushalte auf Basis von Mikrodaten \\ Poor Debtors - Rich Debtors? Household Indebtedness and Financial Assets of Private Households Based on Micro Data}

Pirmin Fessler* und Peter Mooslechner*

\section{Einleitung}

Die Verschuldung privater Haushalte (im Weiteren als Haushalte bezeichnet) hat in den OECD-Ländern langfristig deutlich zugenommen (vgl. Girouard et al. 2006, Barnes und Young 2003). Gleichzeitig stieg jedoch auch das Geldvermögen privater Haushalte markant. Getrennt voneinander haben beide Tendenzen vielfach analytische, aber auch wirtschaftspolitische Aufmerksamkeit gefunden. Simplifiziert wird dabei (medial) immer wieder der Eindruck erweckt, dass Haushalte tendenziell verschuldet sind, wenn (oder weil) sie

* Oesterreichische Nationalbank, Wien. Für wertvolle Kommentare danken die Autoren insbesondere Martin Schürz und Alfred Stiglbauer.

(C) INTERVENTION 5 (I), 2008, 3I-45 